\title{
A Case of Stiffperson Syndrome
}

\author{
Mamdouh H. Kalakatawi, Nusrat Aziz, and Norhan A. K. Mostafa
}

\section{ABSTRACT}

Stiffperson syndrome (SPS) is one of the rarest neurological disorders. It is an autoimmune disease with antibodies against glutamic acid decarboxylase - enzyme needed to produce neurotransmitter GABA. This disease is very debilitating and fatal if not managed in time. A 32-year-old man presented with stiffness of abdominal and thoracolumbar paraspinal muscles first noticed 10 months back. There was history of jerkiness of hands. After excluding other mimicking conditions like multiple sclerosis, parkinsonism and axial dystonia, a diagnosis of Stiffperson syndrome was made. Treatment with IVIG therapy along with anti-spasticity drugs resulted in improved patient outcome. SPS is potentially treatable though cannot be cured. Not one treatment fits all patients with SPS and may have to be tailored according to the clinical manifestations. Increasing awareness in medical doctors and identifying the disease early can help the patients escape the disabling effects of SPS and improve the quality of life.

Keywords: immune disorder, IVIG therapy, rare neurological disorder, Stiffperson syndrome.

\section{INTRODUCTION}

Stiff person syndrome (SPS) is a rare neurological disorder. It was first described by doctors Moersch and Woltman in 1956 in a 49-year-old woman [1]. It has an autoimmune basis with antibodies against glutamic acid decarboxylase-65(GAD-65), an enzyme important for synthesis of the inhibitory neurotransmitter gamma aminobutyric acid [2]. Antibodies can also be found against GABA A receptor protein [3] and glycine alpha-1 receptor [4] and glycine transporter 2 [5]. But $30 \%$ of patients are negative for antibodies. Due to loss of these inhibitory neurotransmitter, there is increased stimulation of brain and spinal cord exhibited as rigidity, spasms of skeletal muscles and even phobias. It is often associated with other autoimmune disorders like Type 1 Diabetes mellitus, pernicious anemia, thyroiditis and vitiligo. SPS has been found to have three presentations 1. Classical SPS, 2. Variant SPS and 3. Paraneoplastic SPS. Antibodies against amphyphysin may be commonly present and antibodies against GAD absent in paraneoplastic SPS [6].

\section{CASE Presentation}

\section{A. Patient Information and Clinical Findings}

A 32-year-old male presented with stiffness of back and abdomen. The symptoms started 10 months back progressing

\author{
Submitted : May 10, 2021 \\ Published : May 31, 2021 \\ ISSN: 2593-8339 \\ DOI: 10.24018 /ejmed.2021.3.3.871 \\ Mamadouh H. Kalakatawi \\ Department of Neurology, Al Noor \\ Specialists Hospital, Makkah, Kingdom \\ of Saudi Arabia. \\ (e-mail: Kalakatawi@yahoo.com) \\ Nusrat Aziz* \\ Department of Physiology, Faculty of \\ Medicine, Umm Al-Qura University, \\ Makkah 24382, Kingdom of Saudi \\ Arabia. \\ (e-mail: drnusrataziz ${ }^{@}$ gmail.com) \\ Norhan A. K. Mostafa \\ Department of Neurology, Al Noor \\ Specialists Hospital, Makkah, Kingdom \\ of Saudi Arabia. \\ (e-mail: norhan.ali1982@hotmail.com) \\ *Corresponding Author
}

to the present condition. The stiffness is continuous. There is history of jerkiness of upper limbs. It has affected his sleep and appetite. The patient finds difficulty in carrying out day to day activities like dressing and sitting straight. He complains of left shoulder pain. There is no difficulty in breathing. The patient does not report any triggering factors. There is no history of diabetes mellitus, hypertension, thyroid disorder, malignancy or any neurological disorder. there is no history of similar problems in family.

On examination the patient was thinly built, conscious, coherent and cooperative. He was ambulant with slow stiff gait. There is stiffness of axial muscles specially the abdominal muscles and the thoracolumbar paraspinal muscles as shown in Fig. 1 A, B, C with decreased lumbar flexion as shown in D of Figure. 1.

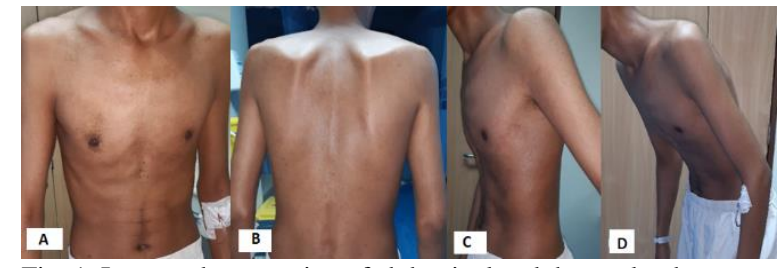

Fig. 1. Increased contraction of abdominal and thoracolumbar paraspinal muscles with decreased lumbar flexion.

On motor examination the power of limb muscles is $4 / 5$. Abdominal, neck and back muscles are firm too hard on palpation. There is no tenderness. The tone of upper and 
lower limb muscles is normal. The deep tendon reflexes and plantar reflex are normal. The sensory system and cranial nerve examinations and test for cerebellar functions are normal. There is no affection of cognitive or higher function. Other systemic examinations are unremarkable.

Vitals noted are temperature $36{ }^{\circ} \mathrm{C}$, respiratory rate 20 per minute, Oxygen saturation $98 \%$ on room air, blood pressure $110 / 70 \mathrm{~mm}$, of $\mathrm{Hg}$, pulse rate $80 \mathrm{bpm}$, pain scale $0 / 10$ at admission. Weight $47 \mathrm{Kgs}$ Height $171 \mathrm{cms}$ and BMI of 16.

\section{B. Assessment:}

Complete blood picture showed hemoglobin 128g/L (130$170)$, red blood cell count $4.1910^{12} / \mathrm{L} \quad\left(4.5-5.5 \quad 10^{12} / \mathrm{L}\right)$ Hematocrit 0.38 L/L (0.4-0.5) MCH, MCHC, RDW Leucocyte count and Mean Platelet volume were within normal range. Coagulation profile, Blood chemistry for liver profile, renal profile, serum electrolytes, lactate dehydrogenase, serum amylase were found unremarkable.

Chest X-Ray was found to be normal. Ultrasound abdomen was unremarkable. MRI showed few tiny cerebral deep white matter nonspecific foci otherwise essentially normal findings.

\section{Therapeutic Intervention}

The patient was admitted in Neurology ward and was started intravenous immunoglobin (IVIG) at a dose of $400 \mathrm{mg} / \mathrm{Kg} /$ day equaling $18 \mathrm{~g}$ (for this patent) given intravenously over 6 hours once a day, Baclofen $20 \mathrm{mg}$ per oral thrice a day and Lorazepam $2 \mathrm{mg}$ per oral twice a day. Trihexyphenidyl HCL (Artane) $2 \mathrm{mg}$, Heparin 5000 IU subcutaneously thrice a day and omeprazole $20 \mathrm{mg}$ once a day were also prescribed. The patient was allowed an attender to assist him. He was given regular diet. Physiotherapy was started on the third day of admission.

\section{Outcome and Follow Up}

By the end of seven days of IVIG the patient was able to sit and walk properly. Still, he was feeling pain in neck on bending forward. The range of motion was increased than prior to therapy. There was overall improvement in patient's functionality. The treatment was well tolerated without any adverse effects observed. He was discharged with medications of baclofen, lorazepam and Artane with advice of IVIG therapy monthly and fortnightly follow up.

\section{DISCUSSION}

The reported incidence of stiffperson syndrome is 1 in $1,000,000$ [7] though doctors think it may be higher. It is mostly misdiagnosed as other neurological disorders and takes an average of 7 years to be diagnosed. It is believed to have autoimmune origin due to presence of antibodies detected in $70 \%$ of cases against GAD-65. Research is still going on and antibodies to other neurotransmitter or their receptors is still being discovered. Scientists are also trying to find out any pathophysiology related to $\mathrm{T}$ cell or $\mathrm{B}$ cell immune system also. Classical Stiffperson syndrome shows axial rigidity which may associated with some rigidity of proximal muscles of lower limb. It can be associated with limb stiffness, epilepsy, dysarthria, abnormal eye movements as in Stiffperson variant syndromes like Progressive encephalomyelitis with rigidity and myoclonus (PERM). Paraneoplastic variant syndromes are mostly associated with breast cancer, Hodgkin's lymphoma, colon cancer lung cancer or malignant thymoma. Paraneoplastic variant show presence antibodies against amphiphysin. Stiffness is increased with stress and sympathetic stimulation. Many patients report about trigger factors. Our patient does say about myoclonus like jerks occurring in hands after washing which progresses further to stiffness in the initial stages of the disease. The age of patients coming with symptoms is 30-60 years with mean age of 42.2 (range 29-57 years). [8] Stiffperson syndrome may be confused with multiple sclerosis, parkinsonism, neuromuscular dystrophies etc vitamin B12 deficiency but a good clinical history and examination can exclude other differential diagnosis. There is simultaneous contraction of agonist and antagonist muscles of abdomen leading to stiff posture and decreased or no lumbar flexion as seen in this patient. EMG testing may show continuous motor end plate potential which indicates Stiffperson syndrome even in absence of antibody testing. This patient showed improvement by treatment with IVIG therapy, GABA agonists, baclofen and physiotherapy. Positive response to IVIG therapy goes in favor of SPS diagnosis. Other treatments like plasmapheresis and Rituximab have also shown to relieve symptoms in other studies. rituximab is a genetically engineered monoclonal antibody against CD20 of B cells and thereby decrease the B cells in blood [9]. Physical therapy is an important adjuvant in treatment, and it is shown to decrease symptoms [10]. It should include deep tissue myofascial techniques, passive range of motions, ultrasound, heat therapy and hydrotherapy. Classical Stiffperson syndrome shows good improvement with physiotherapy. Behavioral cognitive therapy to relax the patient might be helpful to decrease episodes of rigidity by decreasing the sympathetic activity.

\section{CONCLUSION}

Stiffperson syndrome is a painful debilitating condition making a person bedridden which is often fatal. Though this condition is very rare in general population but the impact on the health and quality of life of a person is very devastating. The presentation can vary in different patients. Exclusion of other likelihood conditions and high grade of suspicion for SPS is important to reach diagnosis. Not one treatment can be followed for all patients of SPS and has to be tailor-made according to symptoms and outcome.

\section{REFERENCES}

[1] Moersch FP, Woltman HW. Progressive fluctuating muscular rigidity and spasm ("stiff-man" syndrome); report of a case and some observations in 13 other cases. Proc Staff Meet Mayo Clin. 1956;31(15):421-427.

[2] Baizabal-Carvallo J, Jankovic J. Stiff-person syndrome: insights into a complex autoimmune disorder. Journal of Neurology, Neurosurgery \& Psychiatry. 2014;86(8):840-848

[3] Dalakas MC. Stiff person syndrome: advances in pathogenesis and therapeutic interventions. Curr Treat Options Neurol. 2009;11(2):102 110. doi:10.1007/s11940-009-0013-9.

[4] Alexopoulos H, Akrivou S, Dalakas M. Glycine receptor antibodies in stiff-person syndrome and other GAD-positive CNS disorders. Neurology. 2013;81(22):1962-1964.

[5] Balint B, Bhatia KP. Stiff person syndrome and other immunemediated movement disorders - new insights. Curr Opin Neurol. 2016;29(4):496-506. doi:10.1097/WCO.0000000000000351. 
[6] Geis C, Weishaupt A, Hallermann S, Grünewald B, Wessig C, Wultsch $\mathrm{T}$ et al. Stiff person syndrome-associated autoantibodies to amphiphysin mediate reduced GABAergic inhibition. Brain. 2010;133(11):3166-3180

[7] Stiff Person Syndrome - NORD (National Organization for Rare Disorders) [Internet]. NORD (National Organization for Rare Disorders). 2021 [cited 25 April 2021]. Available from: https://rarediseases.org/rare-diseases/stiff-person-

syndrome/\#: : text=SPS\%20is\%20an\%20extremely\%20rare,individua 1s\%20in\%20the\%20general\%20population.

[8] Bhatti A, Gazali Z. Recent Advances and Review on Treatment of Stiff Person Syndrome in Adults and Pediatric Patients. Cureus. 2015.

[9] Baker M. Treatment of stiff person syndrome with rituximab. Journal of Neurology, Neurosurgery \& Psychiatry. 2005;76(7):999-1001.

[10] Potter K. Physical therapy during in-patient rehabilitation for a patient with Stiff-Person Syndrome. J Neurol Phys Ther. 2006;30(1):28-38. doi:10.1097/01.npt.0000282147.18446.b8. 